\title{
The role of, and the control of, obstruction of the upper aimays and of congenital and other ENT anomalies in excess vertical growth of the face that impede orthodontic treatment
}

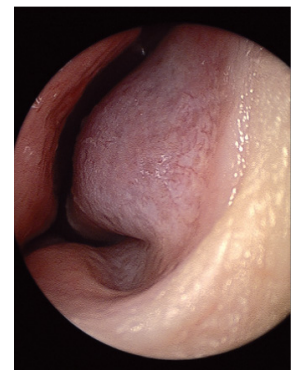

\author{
J. Gilles ABEN-MOHA (ENT), Bruno BONNET \\ (Orthodontist)
}

\section{ABSTRACT}

The objective of this article is to demonstrate the effectiveness of good cooperation between orthodontists and Ear, Nose, and Throat specialists because of the importance of freeing the upper airways from obstruction in order to achieve a good orthodontic result as well as the other side of the coin, the beneficial effect orthodontic treatment can have in stimulating a functional liberation of those airways when no mechanical blockage is present. The article will emphasize how important it is for orthodontists to understand and to be alert to the signs and symptoms that would indicate the need for an ENT evaluation.

\section{KEYWORDS}

\author{
Mechanical and functional obstacles to respiration \\ ENT examination \\ Upper airway problems \\ Sleep apnea syndrome \\ Orthodontic treatment \\ ENT surgery.
}

Address for correspondence: 


\section{1 - INTRODUCTION}

This article is the fruit of a longtime collaboration between two specialists, one in orthodontics and the other in otolaryngology. In it we attempt to present an appreciation of the problems our patients endure and we are called upon to treat, each of us with a specific training and experience in our own fields that color our comprehension of situations we do not routinely encounter in the daily practice of our respective specialties. From the confrontation that our extensive collaboration has forced each of us to make with the special cares and limitations of the other, we have both been granted a golden opportunity to gain a better understanding of the therapeutic indications of our shared enterprise.

\section{2 - THE ORTHODONTIC POINT OF VIEW (DR. B. BONNET)}

When, and after noting what signs, should we ask our patients to seek an evaluation from an otolaryngologist? And at what age? And with respect to what rational protocol?

In some cases the failure to obtain what would have been a critical and redemptive otolaryngological opinion can condemn our orthodontic and orthopedic efforts to treat growth problems, tongue maturation and faulty position difficulties to ineffectiveness and sometimes even harmful outcomes. The ENT specialist, in such instances, can contribute enormously to the success of orthodontic treatment of "facial insufficiency,"

And to an equal extent, "morphogenetically aware" otolaryngologists should be motivated by the same type of reasoning to evaluate the indications for their therapeutic interventions to act as aids in assisting orthodontists to achieve their treatment objectives. In effect, it is essential for all care givers who participate in the effort to correct the growth problems children suffer from to understand the capability of orthodontic/orthopedic mechanisms to prevent or eliminate sleep apnea by clearing the upper airways of obstructions as a secondary benefit of their treatment of "facial insufficiency." The adequate and reasoned intervention of each of the participating specialists can operate effectively only on the foundation of a shared understanding of the formal indications for and feasibility of treatment for growth problems of children.

All of our appliances designed to re-stimulate facial growth aim at encouraging the critical support of an active tongue propelling itself vertically toward the palate but their effectiveness may be compromised or completely negated by obstacles, some of which are otolaryngological in origin, that prevent this upright positioning from occurring.

\section{2 - 1 - Tongue activity confined to a low position: thrust against the cheeks or the lips, or to the chin symphasis}

Orthodontists should never overlook or deprecate the possibility in their 
clinical assessments that patients may maintain their tongues in a low position purely out of habit. And, in fact, in our opinion this behavioral source is the basic reason for adoption of this posture in the immense majority of cases.

In all of these cases the tongue is not held high, not placed vertically but remains stubbornly "hooked," "magnetized," "seduced" by the cheeks (Class I, II, or II division 2), by the lips (Class I or II division 1) symmetrically, or attempting to embrace the chin symphasis. It sometimes happens that the ENT consultation has for its objective not to deprecate this tongue behavioral problem as being the cause of certain malformations but, rather, to ascribe to it, with all delicacy, the full weight of its importance. In fact, the elimination of breathing as a vital etiological factor or pointing to the small contribution it is making are sometimes necessary for setting in motion a simultaneous psychomotor or psychotherapeutic and, if possible familial, support system. The ENT evaluation can help patients and families to reach an understanding that a successful treatment outcome depends largely on the patient's repeated daily response to suggested tongue behavior changes.

\section{2 - 2 - Tongue kept in low position because of anatomic factors: a short, tight lingual frenum}

A short, tight lingual frenum physically prevents the desired posture of tongue elevated, mandible depressed. But in every case orthodontists should guard against the simplistic expectation that a simple lingual frenectomy will stimulate the tongue to achieve an upright position spontaneously. A zone of fibrous tissue often fills the incision and continues to restrict the tongue's free movement. We have found it advisable before surgery to provide patients with a suitable orthodontic device, such as a tongue envelope or a Herbst appliance to help them keep the tongue elevated so that repair tissues forming in the incision will not lock it in its habitual low position.

It is important to note that using the occasion of the general anesthesia employed for an ENT operation on the nasal fossas as an opportunity to add a lingual frenectomy gratuitously may well produce a scar tissue locked tongue owing, doubtlessly, to the temporary post-operative nasal obstruction forcing patients to breath through their mouths, thus leaving their tongues in a low posture.

\section{2 - 3 - Tongue kept in low position when patients are forced into mouth breathing}

We should be careful to differentiate between the various factors that force patients into mouth breathing such as a growth problem in what is called facial insufficiency, from anatomic airway obstructions, because of inflammation, or owing to allergies.

\section{$2-3-1$ - The need to breathe through the mouth because of insufficient facial growth}

All the types of insufficient facial growth can cause constriction of the upper airways. This is well known and 
it is frequently suggested that expansion of the upper arch is a means to overcome this problem. But the possibility of a simultaneous vertical as well as transverse bi-maxillary course of therapeutic action according to the lines of procedure we recommend in this article is often under-estimated and even misunderstood. Its description can be found on line on the www.revue-odf.org site as published in the 2010 issue 4 under the title A Combined Orthodontic and Otolaryngological approach to "facial insufficiency" and hyperdivergence.

\section{2-3-2 - Deviations in tongue posture caused by "anatomic oto-laryngological obstacles"}

- Tongue blocked in a forward position by a large mass of enlarged adenoidal tissue

Sometimes, enormously enlarged adenoids appear on an X-ray film, seeming to force the tongue into a forward position. In such cases, attempts by practitioners to upright the tongue's posture and to move it backward, are, at best, impeded, and in the worst case, totally blocked by the mass of adenoid tissue.

In order to make the indicated orthopedic and functional therapy effective and appropriately swift, it is often necessary for the otolaryngologist to perform a tonsillectomy.

Numerous post-operative cephalometric films demonstrate the re-establishment of correct backward movement of the tongue and an upright positioning of its posterior elements that would not have been possible without surgical removal of the hypertrophied tonsillar tissue that was formerly inducing a nausea reflex.

\section{- Tongue blocked into a low position by obstacles to nasal breathing.}

- Obstacles in the region of the nasal fossas

Sometimes a clinical examination suggests that an obstacle is impeding nasal breathing and accordingly preventing the tongue from assuming an upright position.

Many clinical signs, like pinched nostrils or clouding of the mouth mirror that we use throughout our dental examination, together with information gleaned from the intake interview, as well as views of nasal fossas congested by hypertrophy of the inferior turbinates with or without septal deviations all suggest the advisability of an ENT assessment. Sometimes we evaluate the assessment of the malformations as grave when, for example, there is a tendency to anterior vertical deficiency and lip incompetence and excessive labial inclination of incisor teeth. At other instances we consider ENT intervention less urgent when patients exhibit mixed nasal and mouth breathing during the day as well as at night even after functional orthopedic expansion therapy.

\section{- Obstacles in the rhino-pharyn- geal area}

Sometimes $\mathrm{X}$-ray films show seriously enlarged adenoidal tissue that appears to constitute a blockage of nasal breathing and, accordingly, to prevent the tongue from assuming an elevated position. 
In cases of severe anterior and lateral open bite caused by hyperdivergence with a posterior vertical deficiency as well as an anterior vertical deficiency, an adenoidectomy is the logical and indispensable first step.

\section{3 - THE ROLE OF THE OTOLARYNGOLOGIST (DR. ABEN MOHA)}

Otolaryngologists acquire in their training and in their routine daily practice only an incomplete knowledge of cranio-facial growth and of orthodontic procedures: but this understanding will develop as their collaboration with their orthodontic colleagues develops. In this presentation I shall outline some of the problems posed by our joint endeavors.

\section{3-1 - When should an orthodontist ask for an ENT evaluation?}

Orthodontists refer patients for an otolaryngological assessment, in my experience, after they have noted nasal obstructions, mouth breathing, low tongue position, an anatomic obstruction on an X-ray film, or simply because the orthodontic problem is severe and the need for a complete work-up becomes evident. Additional reasons impelling orthodontists to seek ENT opinions include failure of any of the gamut of myofunctional training, courses, a patient's inability to wear a nocturnal orthodontic appliance, an unsatisfactory orthodontic treatment outcome, an unstable result, and, the beginning of relapse.

\section{3-1-1-Obstruction of the upper nasal and rhino- pharyngeal airways}

Obstructions in the upper nasal airways and in rhino-pharyngeal passages, which are often associated with each other, make mouth breathers out of the children who suffer from them and also oblige them to carry their tongues in a low, anterior position. By thus diminishing tongue action on the maxilla, they are also responsible for a contraction of the upper arch that prevents it from articulating properly with the lower arch, a relationship that provokes an excess of vertical development of the face.

This combination of altered nasal breathing and a preponderance of mouth breathing that prevents some orthodontic patients from thrusting their tongues against their palates may explain their reluctance, even outright refusal, to wear appliances like night guards. In sum, airway obstructions and their consequences may degrade the quality or the stability of an orthodontic treatment result.

\section{3 - 1 - 2 - Macroglossia}

Macroglossia, involving the free portion of the tongue, its base, or auxiliary parts, associated in varying degrees with a short frenum and hypertrophied tonsillar tissue can force the tongue into an anterior position. Its forward thrust during primary swallowing can lead to anterior open bite, an increase in anterior and vertical growth of the mandible, and a tendency toward prognathism. 


\section{$3-1-3-A$ raising and thrusting of the upper lip under a projecting anterior nasal spine}

A raising and thrusting of the upper lip under a projecting anterior nasal spine also encourages lip incompetence that rapidly establishes a vicious circle of functional mouth breathing, even when no airway blockage is present, and thus provokes excess vertical growth of the lower third of the face.

\section{3-1 - 4- Lack of muscular equilibrium}

Similarly, a lack of balance between the tongue musculature and the labial and cheek musculature prevents facial growth from unfolding harmoniously.

Noxious habits like sucking of thumbs and pacifiers can provoke anterior or lateral open bites whose symmetry or asymmetry depends on the choice of which fingers to suck patients make and the intensity with which they suck them. They can also lead to under-development of the maxilla if, for example, a thumb is kept lodged against the hard palate, or cause an upper alveolar protrusion accompanied by a retrusion of the lower alveolar processes or even a retrusion of the mandible itself.

\section{3-1 - 5 - Doubt of blockage}

Sometimes the onset of orthodontic treatment helps some patients who had been mouth breathers to close their lips correctly and begin nasal respiration. For them, especially when there is doubt about the presence of a frank airway blockage, a proposed surgical intervention should be postponed.

\section{3 - 2 - What questions to ask, and things to look for, in the intake interview}

\section{3- 2 - 1 - Breathing}

\section{- During the day:}

- is nasal obstruction unilateral or bilateral?

- is it symmetrical or alternating

- is mouth breathing permanent or temporary? Is it initiated by circumstance or position, lying down, for example, by time of year, a visit to the countryside, or by exposure to allergens?

- is it possible for the patient to breathe through his nose? Or is it impossible? Does patient use mouth breathing exclusively?

- when respiration is primarily by mouth, is nasal breathing uncomfortable?

- can patient maintain nasal breathing? for how long? while playing sports? does patient suffer from episodes of dyspnea?

- is respiration noisy?

- At night: the examiner should ask the same questions with the addition of these:

- is the mouth kept open? Is it dry?

- is saliva left on the pillow by morning?

- does patient wake up at night to get a drink of water? is the mouth dry in the morning?

- are there episodes of apnea? with snoring? and/or with pauses in breathing? 


\section{- Other questions:}

- rhinolalia: does patient speak with a nasal tone owing to excessive closure or openness of a nasal passage,

- does patient suck thumb or a pacifier?

\section{3-2 - 2 - Difficulty in sleeping}

- does patient have trouble falling asleep?

- is his sleep disturbed by agitation, nightmares, shouting, somnambulism?

- does patient snore or breathe noisily?

- are there periods of apnea or pauses during sleep?

- does patient wake with a start or get up for a drink? Is she a bed wetter?

- does patient suffer from night sweats?

- is her morning awakening difficult?

- does patient awaken from a night's sleep feeling refreshed?

\section{3-2 - 3-General health status Repercussions}

- Does patient show any signs of the sleep apnea syndrome? Examiners will find the Epworth index of daytime sleepiness most useful. When the Epworth index suspicion scale is over 10 in older children and adolescents, a sleep polygraph will be helpful.

- Does patient suffer from somnolence or headaches?

- Can patient be described as suffering from asthenia, or generalized weakness, or is she alert with good body tonus and an active participant in sports?

- Is patient able to concentrate and do well academically or does he have learning difficulties?

- Does patient have any memory lapses?

- Is he irritable, nervous, apathetic?

- Does patient have a history of cranio-facial trauma?

\section{3-2-4-Presence of inflammation and infection}

Examiners should look for or check:

- Rhinitis or pharyngitis, snoring, tonsillitis, distal rejection of phlegm indicated, perhaps, by sniffling during swallowing, hemmage, sinus pain.

- Posterior hypopharyngeal and laryngo-tracheo-bronchial reflux, digestion problems.

- Inflammation and extensive reflux: asthma, renal problems, cardiac murmurs, arthralgia

- Otological condition; tympanic condition, tubular catarrhs, hearing problems (an audiogram may be indicated).

- Any known allergies, vaso-motor signs, any impairment of sense of taste or smell, nasal itching. An assessment of allergies, skin, and blood condition is best made in patients more than seven years old when allergens can be effectively discerned.

\section{3- 2 - 5-Presence of accessory problems}

- Some questions to ask. Does patient suck thumb, finger, or pacifier? If so, 
with what intensity and frequency? With which fingers, of which hand? Or has patient recently stopped the habit? The presence of such a habit may explain why an anterior open bite is more marked on one side than the other or why it is centered around a digit placed against the hard palate.

- Does the patient have any swallowing problems?

- Does she eat "nervously" or noisily, jump up from her seat frequently, chew her food badly and swallow it quickly, and have trouble synchronizing breathing with deglutition?

- Does her ingested food sometimes take wrong turns with nasal regurgitation?

- Is there any reflux, vomiting, or nausea?

Has a physician previously examined or treated the child for:

- appetite problems, anorexia?

- speech problems with pronunciation difficulties?

- posture problems: are any positions painful? Does patient have neck or back pain? Are there signs of scoliosis?

\section{3 - 3 - The examination}

\section{3 - 3 - 1 - The initial inspection}

- The facial exam: what the practitioner looks for

- Facial symmetry. A uni-lateral nasal obstruction can aggravate asymmetry.

- Growth, vertical excess of the face, lip incompetence, mouth held open from time to time or at rest. Do muscles contract at the menton tuft when patients try to close their lips?

- Status and position of lips. Is the upper lip short or does it project? Does patient have a gummy smile?

- Is the tongue readily visible? Does patient thrust it between teeth in the initial phase of deglutition? Are the tongue's borders notched? Does the tongue seem large and in constant movement, with quivering at what should be a stable rest position?

- Does patient have any congenital malformations, or jaw retrusion?

- Is there any evidence of malformation of the nasal pyramid or the facial profile that are possible sequellae of trauma or of fractures of facial bones. Is there a puffed-up area below the menton, often a sign of mouth breathing?

- Physicians can assess symmetry of nasal breathing by placing a dental mirror under each nostril to see which clouds it up. They should check to see to see if nasal breathing improves after instillations of vasoconstricting nose drops that may stimulate patients to close their lips.

- The old notion that "adenoidal facies" cause children to breathe exclusively through their mouths has been abandoned for many years.

- What the physician should look for in a global inspection

- What is patient's global posture, inclination of the head, and inclination of the neck?

- At what level are the patient's eyes and shoulders? Are they symmetrical? 
- Are the patient's cervical muscles contracted?

- Is there any sign of tirage, an inspiratory recession of the chest wall that would indicate an obstruction of the larynx?

- Are there any regions of generalized hypertrophy? Is the patient's appetite good or are there indications of anorexia?

- What is the patient's weight and general tonus?

- Does the patient show any signs of psychological distress?

\section{3-3 - 2 - The physical exam}

\section{- Nasal fossas and the cavum}

Physicians make a rhinoscopic examination of the anterior and posterior fossas and, after anesthetizing nasal soft tissues and atomizing them with a vasoconstricting solution, perform an endoscopy that allows them to study the:

- nasal septum and its possible high deviations that could cause pain, headaches, problems of the sinus and of olfaction, and be and be at the root of respiratory difficulties. As a general rule patients prefer to sleep on the side of the septal deviation because the interference with venous blood flow on this homolateral side in patients who are lying down is aggravated even more. They unconsciously protect the better functioning nostril by placing it in the uppermost position;

- volume of the turbinates. Their possible retraction after application of vasoconstrictors may ameliorate respiration sometimes causing patients to indicate this improvement spontaneously by closing their mouths;

- status of inflammation. A pale or lilac color of the mucosa indicates an allergy provoked inflammation;

- presence or absence of polyps or pus at the sinus meatuses;

- status of the senses of smell and taste;

- status of the interior of the sinus cavities, if the ostia are open;

- the patency of the choanae, the posterior nasal passages; the volume of the cavum and the volume of the adenoidal vegetations that may have grown in it; closing of the soft palate during phonation. To test this the physician may ask patients to hold the pronunciation of the letter " $A$ " while they speak and during swallowing.

Contraction of extruded tissue known as Passavent's ridge, when present, constitutes a transverse barrier that aids in closing entry to the cavum.

\section{- The oral cavity}

The physician should look for and make assessments of:

- The patient's orthodontic and dental status noting presence or absence of diastemas or temporary teeth.

- Evidence of bruxism, presence of worn areas on teeth, status of dental articulation. Discoloration of anterior teeth may be a sign of mouth breathing. Physicians should also check the constriction of the masseter muscles and the status of the temperomandibular articulation.

- A thick labial frenum interposed between an upper anterior diastema 
and its possible interference with the orthodontic appliance.

- Mobility of the tongue. Does its bulk suggest macroglossia with its borders notched from imprints of teeth? Is the tongue held in an anterior position? In the first stage of swallowing does it thrust itself between an anterior open bite or a diastema from a low position?

- Does the lingual frenum, which may be short, thick, and bifurcated near the Wharton ducts, prevent the tongue from being raised? If it is tightly attached to the base of the tongue, the frenum may impede the tip of the tongue from advancing, which makes pronunciation of certain sounds difficult and, on rare occasions, because of its lack of propulsive power may send boluses of food on a false route backwards.

- A narrow palate with a retruded maxilla prevents the mandible from being enveloped by the upper jaw and reduces nasal breathing and may also be a sign of excessive thumb sucking.

- Status of the cheek mucosa. Findings might include slightly moist appearance, evidence of bite marks or lichen planus, or of cheek sucking.

- Position and size of the tonsils whose poles may be set in the soft palate in relationship with the columns and may descend steeply toward the base of the tongue where they can be exposed to infection, crypts, and caseum.

- The soft palate and the tonsillar columns, which may be long, thick, and hypertrophied.

- The uvula, which may be long and bifurcated, imitating the appearance of a cleft palate. Palpation may detect the presence of an osseous cleft in the maxilla above it.

- The oropharynx, which may be thickened and show traces of inflammation due, as a rule, to esophageal reflux.

- the diameter of the hypopharynx, with the Muller maneuver. To do this, after asking patients to make a forced expiration, the physician asks them to inspire while holding their mouths and nostrils closed and then uses a fibroscope to obtain the results.

- The size of the base of the tongue and the lingual tonsils with their crypts.

- Additional examinations

- Otological to see if nasopharyngeal obstructions have affected the ears.

- Broncho-pulmonary.

\section{- Para-clinical examinations}

Depending on results of the clinical review, physicians may decide they need the additional information that can be provided by:

- A profile radiological view of the cavum and the sinuses.

- An audiogram.

- A sleep polygraph using the Epworth Index to evaluate the possible presence of the sleep apnea syndrome.

- Functional breathing tests.

\section{3 - 4 - Non-surgical treatment}

\section{3-4 - 1 - Medical}

- This is often required because the fragile tissues of the region are highly susceptible to repeated 
bouts of infection that can become chronic. Patients need to be advised on how to take precautions and how to maintain good preventive health behaviors. Physicians use sonic aerosols, antibiotics, and corticoids to treat pain, sinusitis, and tonsillitis and to stimulate immune responses.

- Physicians deal with esophageal reflux by advising patients on proper eating behavior and, in extreme cases, with surgery.

- Thermal cures are employed for infectious and allergic problems.

- In their treatment of allergies and asthma, physicians may prescribe:

- corticoids for short term benefit,

- anti-histamines in the form of antiallergic nose drops. Corticoids with vasomotor effects can be substituted for purely vaso-constricting noses drops, but only for patients older than 15 as a trial treatment.

- Physicians will eventually deliver more definitive allergic and later specific immune-desensitivity treatment.

\section{3-4-2-Various recommendations and re-training programs}

- Patients have to be taught how to stop sucking thumbs, fingers, or pacifiers. At first they are allowed to fall asleep with the desired object in place, knowing that it will be removed when they are sleeping and that they should not replace it.

- Patients can be asked to voluntarily try to close their mouths and breathe through their noses.

- Re-training:

Speech therapists and physical therapists can help by showing patients how to adopt proper tongue posture and how to make sounds using the correct pronunciation tools and organs, not to speak nasally or to make other dysphonic sounds.

Visits to therapists should produce at least partial results in 10 to 15 appointments. If they do not, physicians should look for a possible mechanical cause of the problem.

\section{3 - 5 - Surgical treatment}

\section{3 - 5- 1 - Surgical procedures}

\section{- On the nasal septum}

The surgeon performs an endoscopic septoplasty (figs. 1 to 3 ) under general anesthesia, sometimes on quite young children when there is a major near total or total obstruction, usually as a result of trauma, even though it is understood that growth and orthopedic expansion of the maxilla may provoke a new deviation that will have to be re-evaluated and may require another surgical procedure.

Surgeons therefore prudently limit their early interventions to severe deviations and scrupulously avoid any adjustment of posterior and superior portions of the nose. This procedure may be carried out in conjunction with an endoscopy of large size and swollen middle turbinate bones thought to be causing pain, headaches, chronic sinusitis from faulty aeration, or olfaction problem.

\section{- Operations on the inferior turbi- nates (fig. 4)}

It is often advisable for surgeons to reduce the size of the inferior turbinates, under local or general anesthesia, but never to remove them 


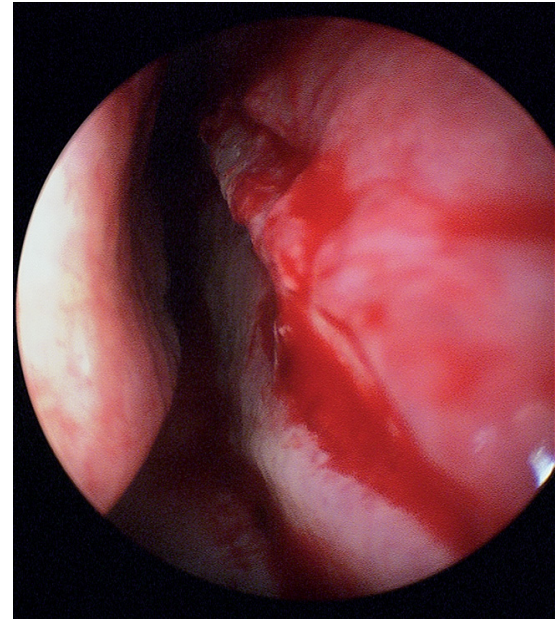

Figure 1

A deviated septum.

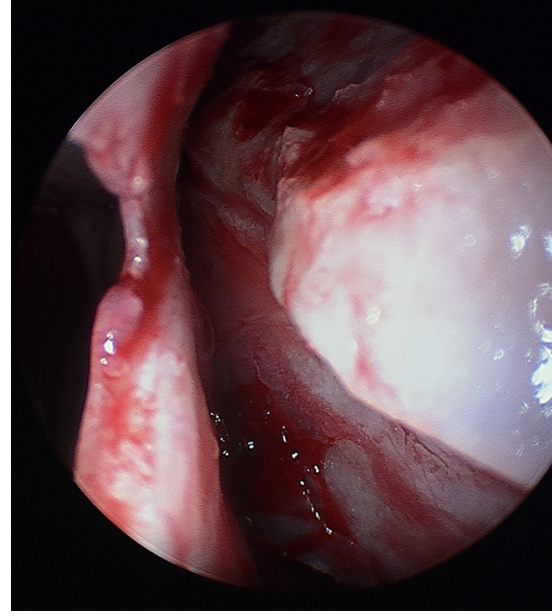

Figure 2

A separated septum.

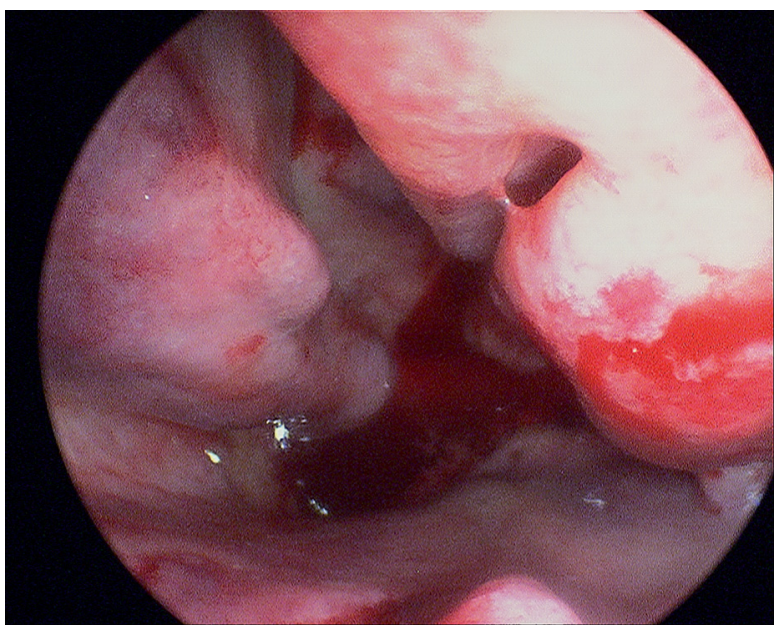

Figure 3

A septal deviation and the inferior turbinate bone.

entirely. The amount of osseous tissue removed depends upon the age of the patient and the size of bones. This operation often eliminates the necessity of correcting a mildly deviated septum.

Surgeons accomplish this procedure either:

- by thermal cauterization or by radio frequency ablation in endoscopy or with a laser under fibroscopy;
- by electrocoagulation with a bipolar pliers under general anesthesia (fig. 5);

- by luxation with aid of a Kilian speculum (figs. 6 and 7), a procedure that may also be associated with electrocoagulation;

- or by a partial turbinectomy (fig. 8) that reduces the bone's height and, usually, leaves the base mostly unaffected to lower the risk of 


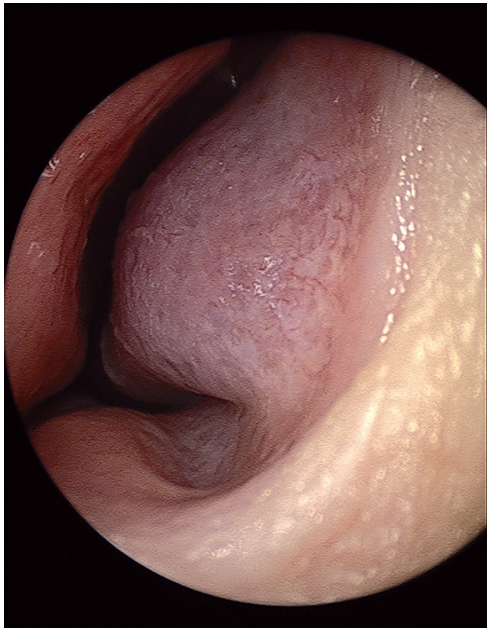

Figure 4

Hypertrophied lower left turbinate.

hemorrhage. A portion of the osseous skeleton of the turbinate may be resected, possibly asymmetrically.

After completing procedures on the inferior turbinates under general anesthesia, surgeons usually interpose a silicone plaque between them and the septum for 15 days to make certain no adhesions develop between these two structures.

\section{- Adenoidal vegetation (figs. 9 and 10)}

Surgeons can remove these either by:

- a traditional adenoidectomy passing through the mouth under the soft palate, if the adenoidal tissue is to be found in the cavum, its usual position,

- or under control of endoscopy through a combined oral and nasal approach if the adenoidal tissues had invaded the posterior choanae of the nose where a rhino-pharyn-

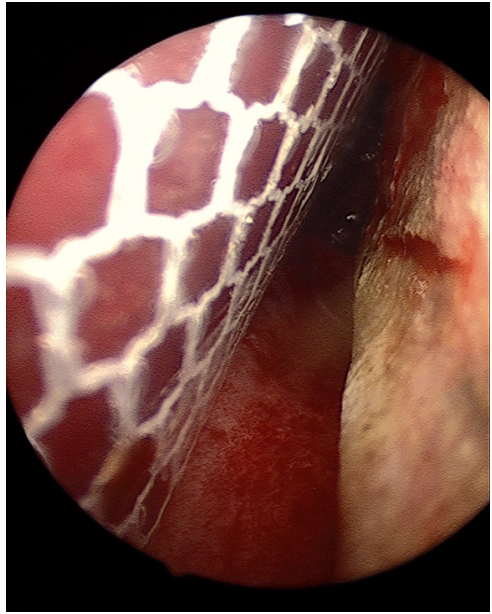

Figure 5

Electrocoagulation of the lower left turbinate with interposition of a silicone plate.

geal approach would not reach them.

For older children and adolescents, physicians should regard the adenoidal tissue of their patients with vigilance conducting frequent anatamo-pathological examinations as a matter of principal, because this tissue can disappear spontaneously or relapse into its former state as a result of esophageal reflux.

Whenever a child is placed under general anesthesia for another surgical procedure, surgeons usually take advantage of the opportunity to remove any residual adenoidal tissue even if only a small amount of it is left.

\section{- Frenums}

- The lingual frenum (figs 11 to 15)

A surgeon performs a lingual frenectomy by resecting the hypertrophied portion on the tongue's ventral surface being careful not to impinge upon the nearby Wharton submandibular ducts. The surgeon frees the lateral borders and places an inverted 


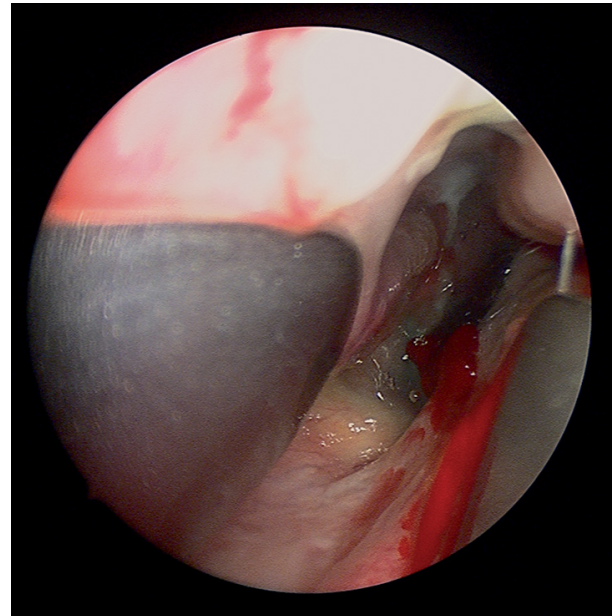

Figure 6

Luxation of the lower left turbinate.

transverse suture in a forcible effort to prevent relapse, always bearing in mind the possibility of provoking growth of taut scar tissue that would mimic the original condition. A tongue carried in a low position and chronic mouth breathing encourage this undesirable outcome.

- Sectioning of upper and lower labial frenums is far less frequently indicated

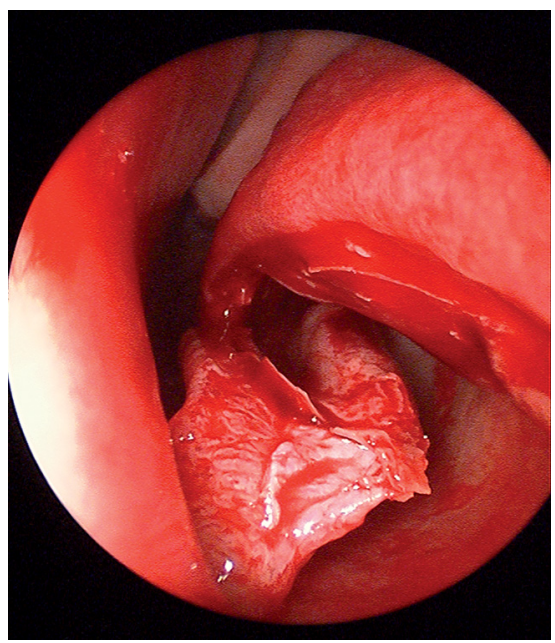

Figure 8

Partial lower left turbinectomy.

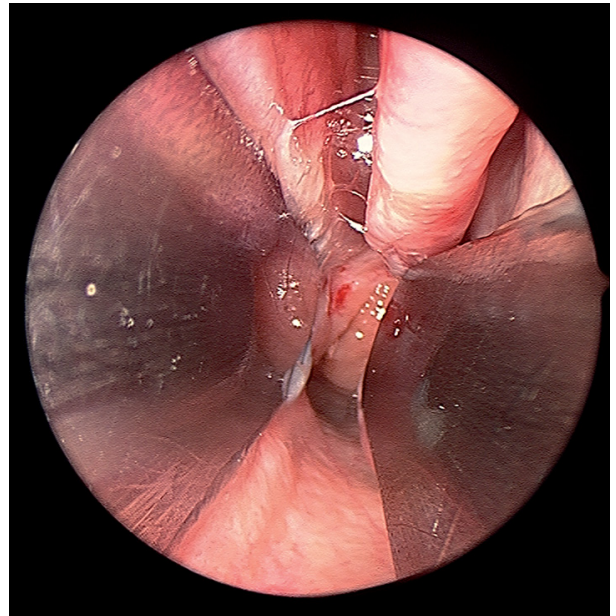

Figure 7

After luxation of lower left turbinate, hypertrophied adenoidal tissue and middle cornet can be seen.

\section{- Tonsillectomies (Figs 16 to 18)}

Always bilateral even in patients displaying size asymmetry, hypertrophied tonsils are removed by dissection under general anesthesia with surgeons maintaining effective hemostasis and carefully avoiding cutting into

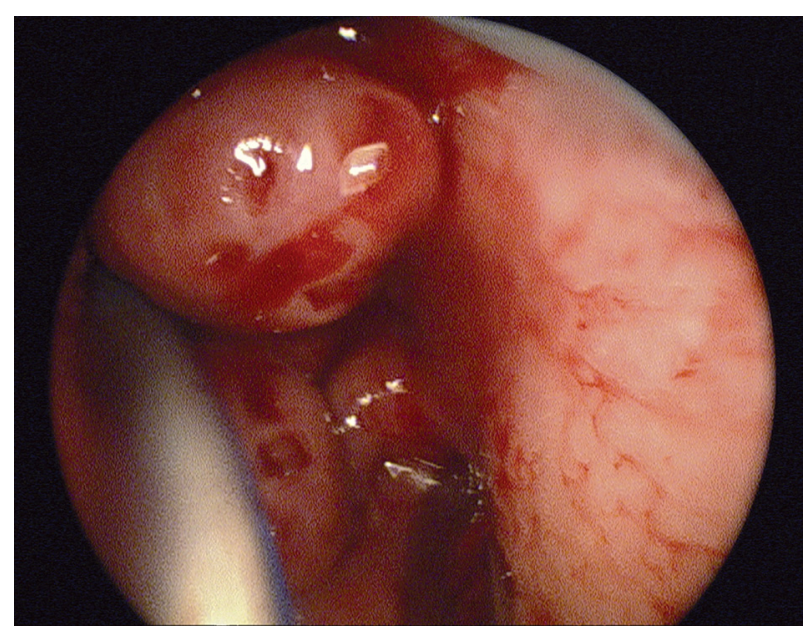

Figure 9

Hypertrophied adenoidal tissue. 


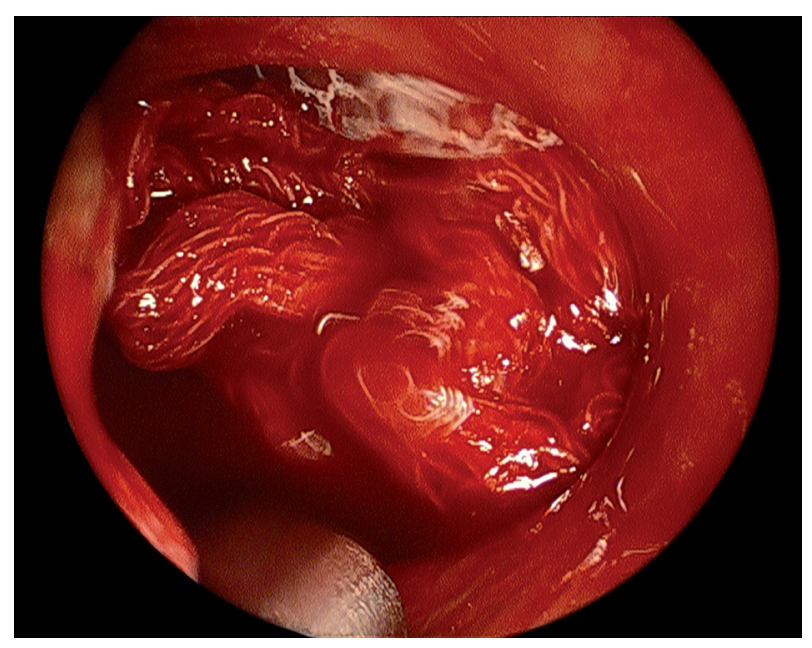

Figure 10

After an adenoidectomy and clearing of the posterior choanae, a compress has been placed in the cavum to plug it up.

the pillars especially in young children to protect against the potential risk such a misstep would pose of deforming the soft palate with accompanying disturbance of phonation and installation of open rhinolalia. Such a deformity would also impair the digestive process by causing false alimentary routes and nasal regurgitations if the posterior pillars were pulled forward. Even worse would be a shortening of the soft palate that might occur if a removal of overabundant adenoidal tissue were attempted at the same time.

- Partial anterior glossectomies are performed only in cases of enormous hypertrophy of lingual tissue.

- Surgeons may also be called upon to undertake certain associated otological interventions, creating trans-tympanic aerators, for example, and to perform endoscopic examination of sinuses, as well, because disturbances in this region often accompany respiratory insufficiency problems caused by anatomic and organic obstructions.

\section{3 - 5 - 2 - Indications for surgery}

It is imperative for practitioners to evaluate every possible surgical intervention with extreme care, in terms of the risk/benefit ratio, evaluating all relevant information particularly about the possibility of complications, even though they are extremely rare. But when they do occur, they may pose serious health threats, even life threatening ones, like hemorrhage. They may require surgical re-intervention that could be of an urgent nature. Before undertaking any operative procedure surgeons should carefully explain its goals, its probable results, and its potential benefits, as well as its risks, to patients and parents in order to obtain their informed consent and cooperation.

\section{Surgeons make their final deci- sion to operate only after:}

- a period of alternative medical treatment has convinced reluctant patients how beneficial a proposed operation would be. Surgery should be postponed if any alternative medical treatment would improve conditions. Then if this alternative approach fails or provides insufficient improvement, surgery can be undertaken;

- orthodontic treatment of facial deformities has accomplished as much as it can and surgical intervention is needed to complete treatment or stabilize it;

- the prospective patient has reached the age and stature 


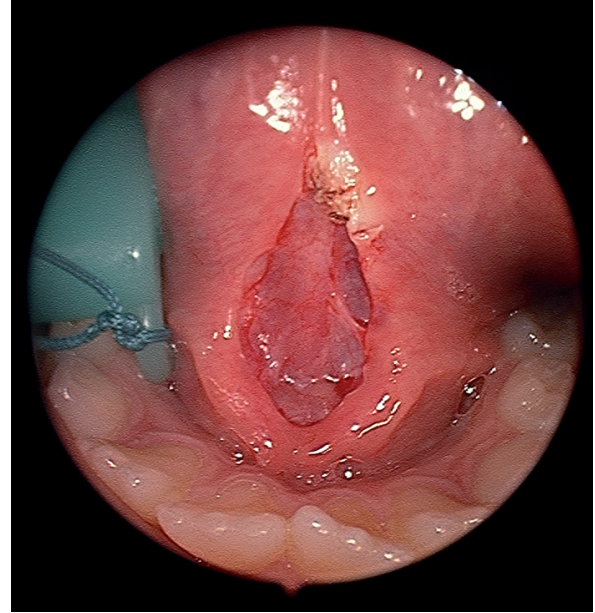

Figure 11

A simple section of the lingual frenum.

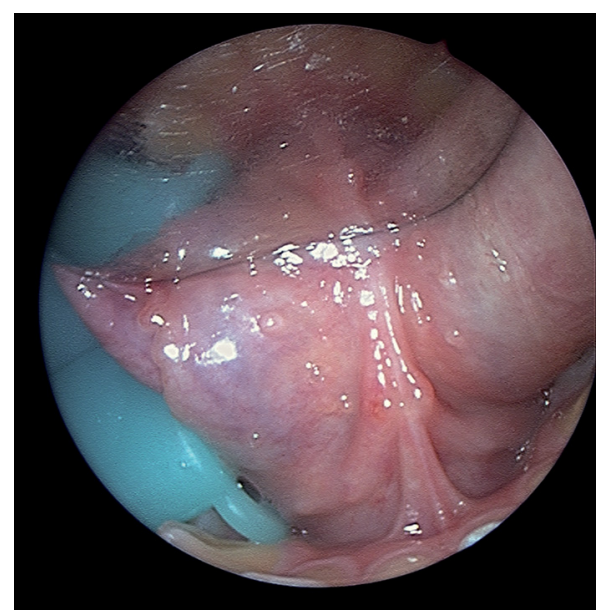

Figure 13

A bifurcated lingual frenum, Wharton duct, and bite block.

considered desirable for the intervention; but the presence of major problems may persuade surgeons of the necessity of operating on very young children;

- it has become clear that myofunctional therapy for respiration and

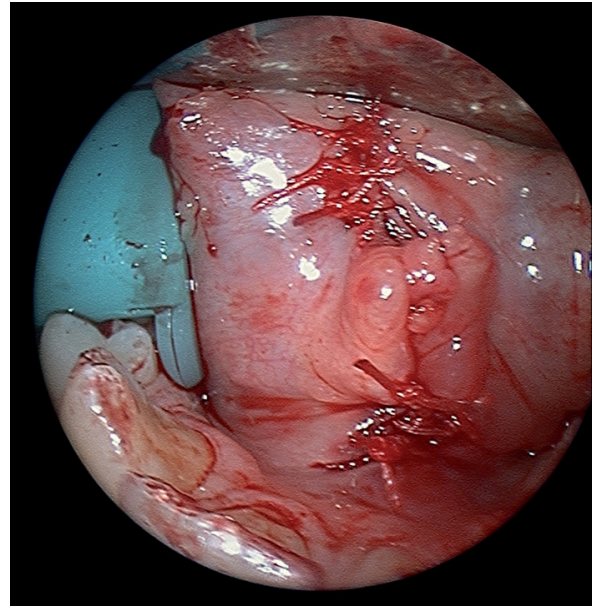

Figure 12

Suture placed in the lingual frenum.

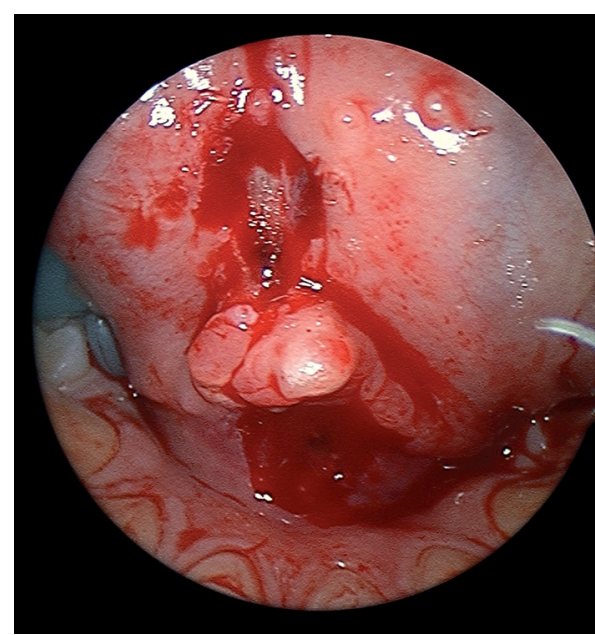

Figure 14

Section of the two parts of a lingual frenum.

tongue posture have not yet achieved their objectives in spite of the patient's good cooperation or if orthodontic therapy had not fully attained the desired results; 


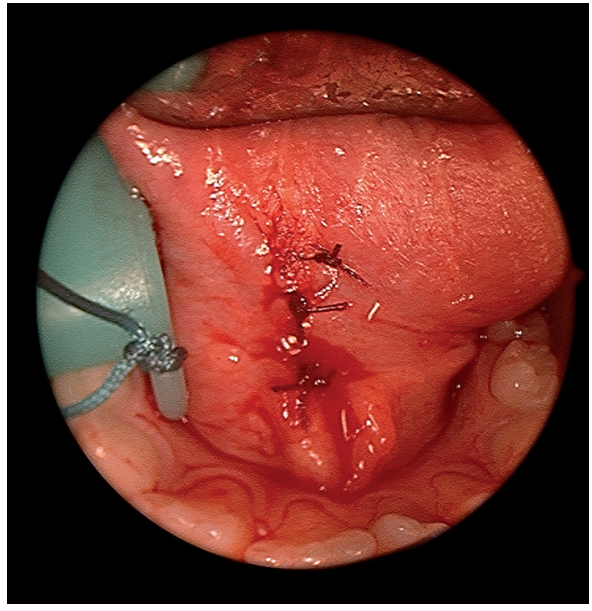

Figure 15

Simple suture in lingual frenum.

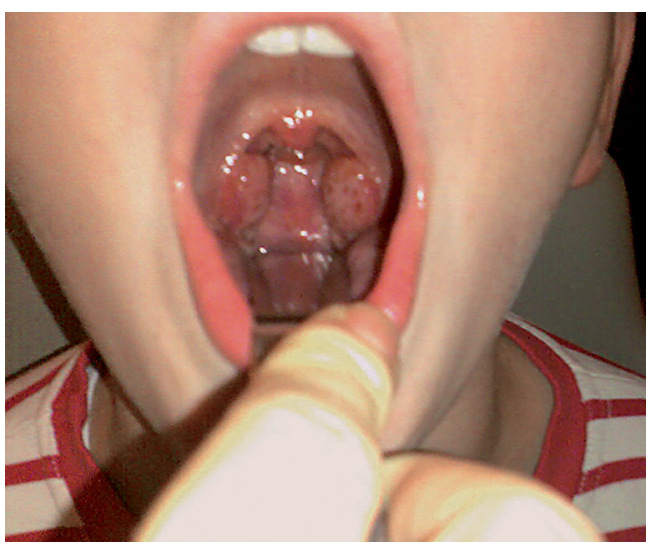

Figure 16

Hypertrophied tonsillar tissue.

- pathological processes, polyposis, for example, are affecting the otological tubes or the sinuses;

- all of the above factors may be added, in varying degrees, to breathing problems, infections, poor general health status, including poor sleeping with its repercussions on waking behavior, loss of

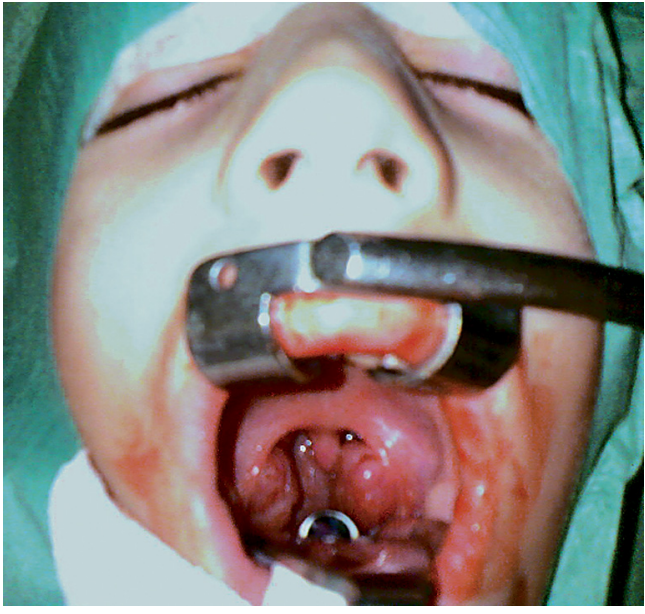

Figure 17

Patient prepared for operation.

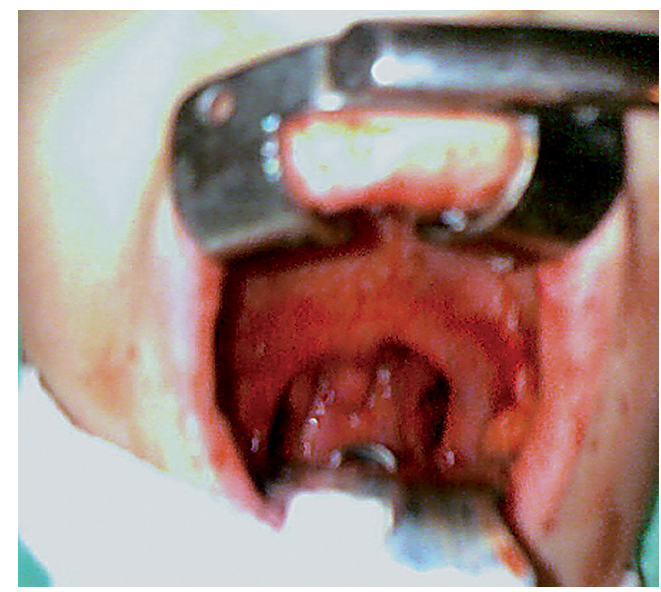

Figure 18

After the tonsillectomy.

appetite, weight loss, and problems in growth and in school.

In order for orthodontic treatment to conclude with a satisfactory outcome patients and parents must offer orthodontic therapists their whole-hearted cooperation. But older and more physically mature patients may become impatient with the apparent slowness of traditional orthodontic treatment 
and favor the "more aggressive" approach that surgery promises.

\section{3 - 5- 3-Over all, contra- indications for surgery depend upon:}

- The results of a careful assessment of the risk/benefit ratio with regard to:

- the presence of a deficiency in blood clotting;

- the possibility of causing a malformation, especially one in the vascular system;

- the possibility of soft palate functioning becoming overloaded if all the airways were to be fully liberated by clearing of obstacles due to vegetations, tonsillar and adenoidal hypertrophy, and blockage in the turbinates. In the face of such an eventuality, even though the formal indications for surgery are positive for a variety of reasons, the surgeon may decide to divide the intervention into two stages, separated by an appropriate period of time, so that the patient can progressively adapt to the new conditions, perhaps assisted by myo-functional and speech therapy. Surgeons may even decide to postpone intervention indefinitely until patients are fully prepared for it, from

\section{4 - CONCLUSION}

We have reviewed some of the unfavorable organic and functional conditions that orthodontists find in their patients at the outset of their treatment and that develop unfavor- orthodontic, growth, and emotional points of view.

- Finally, with very young patients especially, surgeons making decisions based on widely discordant data might be well advised to adopt a posture of watchful waiting to gain time for more rigorous observations, further testing, and, perhaps, to allow orthodontic/ orthopedic treatment to begin and perhaps ameliorate conditions and justify a re-evaluation of the surgical prospects.

- Non-cooperation, even defiance from some families, constitutes, within the highly litigious framework of our present society, more than enough reason for surgeons to postpone, more or less definitively, any intervention.

- Post-operative vigilance, designed to avoid or at least limit complications, like nasal adhesions is crucial. During this period, re-training patients in proper nasal breathing, affected by the palate and the tubules, may be indicated.

In any case, after an initial evaluation for possible surgery, surgeons should see all patients for at least one more visit, with the possible exception of those whose first assessment ruled out any surgery at any time.

ably as treatment proceeds. In many cases, otolaryngologists are called upon to review the status of such patients and to establish a well-reasoned risk/benefit ratio for a 
possible surgical procedure. When the inter-disciplinary team decides to postpone a surgical intervention, the ENT specialist will later re-evaluate the situation taking into account all the contra-indications and dangers that might accompany an operation designed to help optimize the orthodontic/orthopedic result, one that would not, in any case, rule out the possibility of orthognathic surgery at a later date. 